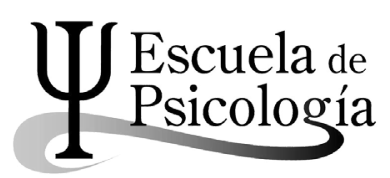

Wímb lu, Rev. electrónica de estudiantes Esc. de psicología, Univ. de Costa Rica. 10 (1): 29-46, 2015 / ISSN: 1659-2107

\title{
ARTETERAPIA EN COSTA RICA: ALGUNAS PINCELADAS
}

Art Therapy in Costa Rica: some brushstrokes

\author{
Maricris Castillo Hellmund*
}

Resumen: Este documento tiene como objetivos principales, relatar algunos antecedentes históricos relevantes y referirse a ciertos/as precursores claves del quehacer arteterapéutico en Costa Rica. Dada la falta de documentación escrita sobre este tema, los datos se recopilan de forma oral (entrevistas personales, telefónicas y vía correo electrónico) por lo que se convierten en la principal fuente de información para la elaboración de este trabajo.

Palabras clave: arteterapia, antecedentes históricos, expresión artística.

Abstract: The main objectives of this paper are to relate some relevant historical information and to talk about professionals who contribute in the progress and development of Art Therapy practices in Costa Rica. However, given the lack of written documentation on this subject, the data must be collected orally (ie: personal interviews, telephone calls and e-mails), making this, the primary source of information for the preparation of this work.

Key Words: art therapy, historical information, artistic expression.

PROEDUCA. Costa Rica.

Correo electrónico: crismari20@gmail.com.

Recepción: 26/09/2014 Aceptación: 15/01/2015 


\section{Pinceladas iniciales}

El presente artículo es un esfuerzo para contribuir con el avance de la arteterapia en territorio nacional. Si bien es cierto que Costa Rica es un país joven en la materia, también lo es, que existen diferentes personas que trabajan y realizan contribuciones para darle a esta disciplina un espacio en el pensamiento de los/as costarricenses.

Nuevas generaciones de psicólogos/as, así como profesionales de otras carreras, se interesan cada vez con mayor fuerza en la arteterapia; promueven, así el reconocimiento formal de este campo de conocimiento y actuación en diferentes espacios. La investigación y la documentación escrita se convierten en ejes esenciales en este empuje, especialmente cuando todavía no existen otros documentos científicos que sinteticen lo que se ha hecho. Este trabajo presenta a continuación, detalles importantes de la historia de la arteterapia en diferentes partes del mundo y épocas; se visibilizan, además, nombres de profesionales, aportes y proyectos que giran en torno a la temática en Costa Rica y se plantean interrogantes iniciales con el objetivo de abrir futuros espacios de discusiónreflexión. El contexto costarricense se convierte en un atelier, un espacio para crear y borrar, para romper, rasgar, diseñar y probar. Las pinceladas fluyen y las ideas también.

\section{Bocetos conceptuales: ¿Qué es arteterapia?}

Peral Jiménez (2011) al reflexionar sobre el libro El arte y la persona. Arteterapia: esa hierbita verde escrito por Mercedes Gysin y Mónica Sorín (2011) afirma:

Qué mejor que utilizar la metáfora y lo simbólico para hablar de esta disciplina, comenzando por el título, evocador, intrigante al principio y enormemente acertado al definir el arteterapia como esa hierbita verde que es capaz de abrirse camino en las piedras y las tierras áridas. El arteterapia, para Sorín, construye espacios de resistencia. Es como una hoja lanzada al viento: resistencia contra la indiferencia, el vacío de los sentidos, contra la frivolidad. Intentamos transformar y transformarnos, reconstruirnos y encontrar nuevos sentidos (p.311).

Las diversas asociaciones latinoamericanas de arteterapeutas también hacen sus propuestas. En la Asociación Chilena de Arte Terapia (ACAT), en su página web, explican que la arteterapia "es una especialización profesional de servicio humano, que utiliza diversos medios artísticos y los procesos creativos, con fines terapéuticos, educativos, de prevención, rehabilitación y desarrollo personal" ("Qué es el Arte Terapia," 2014). Bonilla Elizondo (2012), al referirse a algunas prácticas sociales del arte contemporáneo menciona: 
La arte-terapia también debe tener como un objetivo primordial afectar positivamente a aquellos que en un principio no necesitan terapia; en ese sentido, el arte-terapia debe ser una herramienta para producir conciencia, cambio social y una mayor integración de las poblaciones con conflictos psicológicos y sanitarios en el entorno social cotidiano. Por ello es preferible definir el arte-terapia como "una disciplina que utiliza el arte como medio de comunicación, para brindarle a cualquier persona otros canales expresivos y creativos que fortalezcan su desarrollo personal y social y que puede ser aplicado a diferentes ámbitos sociales: centros educativos, centros de rehabilitación, hogares de ancianos, hospitales, casas de acogidos, entre otros" (p. 6).

Entonces: ¿Por qué arteterapia? ¿Por qué se piensa en la posibilidad de realizar ciertos trabajos a través del arte? Caruso Galletta (2008) responde con esta profunda reflexión:

Porque estamos en un contexto histórico y cultural que niega cada vez más lo individual con la ilusión del individualismo, provocando niveles de insatisfacción cada vez más altos, donde las emociones son un estorbo del cual hay que desprenderse lo más rápido posible. Porque la cantidad, calidad y velocidad de los cambios impuestos y no deseados provocan desarraigo e incertidumbre crecientes.

Se pretende, en forma inútil, controlar ese "malestar en la cultura" con psicofármacos. Se nos retacea la posibilidad de reflexionar seriamente sobre conflictos y problemas de nuestra realidad, expresándonos y compartiéndolos con otros para encontrarle soluciones. Se pretende "entretenernos" o "distraernos" de ellos "pelotudeando por un sueño" o "espiando en la casa del gran hermano", para constatar la pequeñez humana donde los excluidos votan para excluir a otros. No hay espacio social para hablar y ser escuchado. La sensación es que es inútil hablar. Por eso es cada vez más necesario el arte. Es preciso comunicar contenidos emocionales. No se puede negar la condición humana sin un costo altísimo que se manifiesta en sufrimiento, enfermedad, locura y muerte (p.228).

No se trata de pensar en la arteterapia como una herramienta total y ya acabada; no hay teoría o escuela que lo explique o lo pueda todo (Caruso Galletta, 2008). Autores como el citado, defienden que no se trata de magia o esoterismo; un ejemplo lo brinda al argumentar que ya las neurociencias permiten explicar científicamente cómo y por qué el arte cura, a través del funcionamiento en red de los sistemas neurovegetativo, endocrino e inmunitario. Por ello, menciona:

$Y$ en esto no hacemos sino recuperar y actualizar, en forma científica y ya no empírica, el conocimiento milenario que fue sedimentado en el desarrollo de la cultura humana y que ha sido ignorado por el positivismo, tan embelesado por la técnica que equipara a los seres humanos con las máquinas (p.229).

Ahora, ¿para ser considerada la arteterapia como tal, tendrá que estar siempre ligada a las nociones de verdad y de conocimiento verificado? ¿Qué sucede con aquellos aspectos del arte que no pueden explicarse desde el ámbito científico? En sus reflexiones, Adorno (1970) escribió: "Es evidente que ya nada referente al arte es evidente, ni en él mismo, ni en su relación con la totalidad, ni 
siquiera en su derecho a la existencia" (p.19) ¿Si el arte ha perdido su evidencia, cómo congeniar el uso terapéutico de su expresión con ese deseo de encontrar certezas? El arte puede honrar las preguntas y, sin embargo, la arteterapia es una posibilidad de integración entre arte y ciencia ¿Por qué negar la expresión artística como un sistema de comunicación válido para entender malestares, terapéuticas y posibles formas de prevención, si el arte antecedió a la ciencia en la historia de la humanidad?

Sobre el tema, Caruso Galletta (2011) amplía:

El arte precedió a la ciencia en la historia de la humanidad. Otra manera de expresar esto, de acuerdo a teorías actuales, es decir que el código analógico de comunicación (aquel en el cual el símbolo que representa acuerda una relación de forma -analogía- con el objeto representado) precedió al digital (el símbolo es totalmente arbitrario y producto de una convención aceptada, sin ninguna relación con el objeto que representa.) Para ser más claros, $25.000 \mathrm{~A}$. de C., para representar la maternidad, se "hacía" un cuerpo de mujer (Venus de Willendorf), para representar un oso se pintaba un oso (pinturas rupestres de las cuevas de Ardeche). En cambio, 3000 A. de C., los caracteres cuneiformes mesopotámicos son arbitrarios y su forma nada tiene que ver con la forma de las ovejas vendidas

o la cantidad de dinero pagado (p. 41).

Agrega este autor que el arte, como el lenguaje más antiguo y diferente al de la ciencia, "puede auxiliarla para 'decodificar', comprender y enviar mensajes; de este modo, permitir la comunicación y resolver algunos problemas" (p. 45).

\section{Una mirada en el tiempo y en otros espacios}

La utilización de la expresión artística como instrumento terapéutico y catártico es antigua y universal. Judith Aron Rubin (1998) explica cómo las sociedades primitivas emplearon diferentes formas de arte en sus rituales de sanación. Utilizaron el canto, el movimiento de la danza y los ritmos de los tambores. Para Rubin, los dibujos rupestres efectuados en las cavernas por los artistas africanos que tallaron máscaras para rituales o los mandalas de arena creados por los tibetanos, son algunas referencias de antecedentes antiguos de la arteterapia moderna. Caruso Galletta (2011) refiriéndose a la antigüedad de la expresión artística como medio de comunicación, menciona: "Las primeras formas de registrar distintas expresiones de la comunicación humana fueron las artísticas, por medio de objetos, estatuillas, o pinturas rupestres que preceden, en aproximadamente 65.000 años, a la aparición de la comunicación escrita" (p.40). Paradójicamente joven, la arteterapia surge formalmente como disciplina en la primera mitad del siglo XX. El autor citado (2008), explica:

No es casual que la terapia a través del arte se haya desarrollado, en la cultura occidental, luego de las dos guerras mundiales. Es cuando el nivel de agresión y 
destrucción de unos seres humanos contra otros alcanzó su apogeo. Es cuando no había palabras o las palabras no alcanzaban para expresar tal nivel de destrucción y pérdidas. Y cuando las palabras no alcanzan ahí está el arte. Aún en las condiciones más adversas, en el límite con la muerte, el arte ayudó a sostener la esperanza y a sobrevivir. En Auschwitz hubo dibujos, pinturas y poemas. En la ESMA hubo regalos de fin de año clandestinos hechos con recortes de diarios; piezas de ajedrez confeccionados con papel, para jugar a escondidas. Theodor Adorno se preguntaba si era posible la poesía después de Auschwitz. No sólo es posible iMás que nunca es deseable y necesaria (p. 228)!

Rubin (1998) señala otros acontecimientos que también influyeron en el auge de este campo de conocimiento, entre ellos: la propuesta freudiana del inconsciente, el interés de algunos/as psiquiatras por los trabajos de arte de sus pacientes, la formación de distintos fórums interdisciplinarios interesados en el arte y las psicopatologías, la aparición de las pruebas proyectivas en el campo de la psicología clínica, el trabajo de algunos/as artistas en hospitales y el interés de ciertos/as educadores/as por una educación artística más libre en las escuelas. Este conjunto de hechos confluyeron, facilitando para la década de los cuarentas, la emergencia y el desarrollo posterior de la arteterapia (Rubin, 1998).

De acuerdo con Susan Hogan (2001), el término "Art Therapy" fue acuñado en 1942 por el artista británico Adrian Hill (1895-1977). En 1938 Hill estuvo internado en un sanatorio al padecer de tuberculosis. Durante este tiempo, tras haberse mantenido dibujando, Hill descubre que el proceso de creación artística le era útil en su propia recuperación. Después se dedicó a promover el método en los hospitales, publicó libros sobre el tema y logró el apoyo de instituciones como la British Red Cross Society y la National Association for the Prevention of Tuberculosis (Hogan, 2001).

En Estados Unidos, Margaret Naumburg (1890-1983) y Edith Kramer (19162014) desarrollaron una labor pionera. Naumburg fundó la Walden School of New York City y desarrolló su Dinamically Oriented Art Therapy; ella veía en el arte, al igual que en los sueños, una forma de discurso simbólico que provenía del inconsciente. Asumía que las creaciones artísticas podían ser comprendidas a través de la asociación libre, respetando siempre la interpretación de cada persona sobre su propio trabajo. Kramer fue una artista vienesa que llegó a Norteamérica justo antes de iniciar la Segunda Guerra Mundial. Pensaba en el arte como un camino para llegar a la sublimación y creía en el poder curativo del proceso creativo. Al igual que Naumburg, Kramer trabajó en el espacio educativo y colaboró en la publicación de literatura sobre arteterapia.

Otras actoras y actores fueron Florence Cane, Victor Lowenfeld, Mary Huntoon, Elinor Ulman, Don Jones, Hanna Yaxa Kwaitkowska, Janie Rhyne, Robert Ault, Myra Levick, Arthur Robbins, Helen Landgarten, Harriet Wadeson, Shaun Mcniff y Mickie McGraw. Educadores/as, artistas, psicoanalistas, investigadoras e investigadores que creyeron en esta forma de trabajo (Rubin, 1998). 
Farias (2007) describe la arteterapia como una disciplina transdisciplinaria, en el sentido de que se va nutriendo de otras disciplinas. La lista anterior ejemplifica esta afirmación, aportando una serie de nombres de personas que hicieron contribuciones desde distintos campos. En el presente documento se sintetizan también los trabajos realizados por diversos/as profesionales, en este caso, en el contexto costarricense. Muchos de ellos/as son psicólogos/as, pero también artistas, médicos, trabajadores/as sociales, musicoterapeutas, terapeutas de lenguaje y ocupacionales, que han encontrado en el arte, una herramienta terapéutica, educativa, de estimulación y/o rehabilitación útil en sus labores. ¿Es la arteterapia un espacio de encuentro multidisciplinario? ¿Por qué la importancia y la necesidad de la interdisciplina? Bonilla Elizondo (2012) comenta:

Efland proclama la necesidad de articular una confluencia armoniosa entre las artes, las humanidades y las ciencias para una educación integral del sujeto. Efland señala la necesidad de reconocer las diferencias que las separan reconociendo las virtudes que las constituyen y que las afirman como complementarias (p.6).

Bulgarelli Mora, una de las impulsoras de la arteterapia en Costa Rica, afirma que en Latinoamérica, países como Argentina, Chile, México, Colombia y Brasil, han sobresalido por sus avances en la materia (A. Bulgarelli Mora, comunicación personal, 21 de marzo de 2014). A continuación se presenta una muy breve descripción de antecedentes históricos de dos de estos países para ilustrar: Brasil y Chile. En el caso del primero, siguiendo a Ciornai y Diniz (2008), la arteterapia tiene sus precursores en las décadas de 1920 y 1940. Osorio César, psiquiatra y crítico del arte, utilizó la creación artística como recurso terapéutico en el hospital psiquiátrico de Juquery Sâo Paulo; mientras que Nise da Silveira creó talleres de arte en el hospital de Engenho de Dentro Rio de Janeiro. La arteterapia comienza a definirse como campo de conocimiento y actuación en los años sesenta, cuando Hanna Yaxa Kwaitkowska regresa a Brasil.

En Chile, según Serrano Morales (2008), la historia surge a mediados del siglo XX. Artistas y profesionales de la salud mental crearon pequeñas iniciativas que buscaron darle al arte un valor como agente terapéutico. Una de estas experiencias se realizó, durante más de 25 años, en el Hospital Salvador. La gestora fue Mimi Marinovic, quien unió el psicodrama con diferentes actividades plásticas, creando una intervención que no sólo afectó a pacientes, sino también a los profesionales de la salud y a los estudiantes que asistían (Serrano Morales, 2008). Cada lugar tiene sus propias historias que narrar, y, aunque en ocasiones es difícil hablar de arteterapia porque aún es una disciplina muy joven, son muchos los esfuerzos que se vienen realizando en distintas partes del mundo, para darle a esta profesión un lugar dentro del pensamiento de las personas (p.18). Caruso Galletta (2008) indica: 
Conocemos y valoramos a quienes nos precedieron en esta tarea en nuestro país, algunos hace casi cien años, aunque no se tuviera en aquella época la conciencia de la importancia de esos emprendimientos aislados ni se los llamara arteterapia. Pero fueron abriendo un camino que cada vez ha sido más y más transitado (p.228).

Es probable que algo similar haya sucedido en Costa Rica ¿Cuántos/as artistas, psicólogos, médicos, etc. habrán incluido desde hace mucho tiempo, la expresión artística como una herramienta terapéutica, educativa o de estimulación en sus quehaceres? Pérez Yglesias (2012) por ejemplo, plantea que "el polifacético artista plástico José Francisco Alvarado Abella (1929-2001) se destaca como gestor cultural, profesor, pionero en arte-terapia y reciclaje artístico y ecológico..." (p.1). También hay ejemplos de varias instituciones en el país, que desde hace varias décadas vienen desarrollando y utilizando esta disciplina en sus talleres ocupacionales. La arteterapia va más allá de la expresión a través del arte; es un campo de conocimiento y actuación, que según indica Caruso Galletta (2008) "para emplearla hay que saber tanto de arte como de terapia" (p.225).

\section{Bulgarelli Mora: sus primeros trazos}

En 1988, luego de dos años de estar realizando estudios de posgrado en Norwich University, ciudad de Montpelier, Vermont Estados Unidos, Ángela Bulgarelli Mora regresa a Costa Rica. Su retorno y su trabajo representan un florecimiento importante para la arteterapia en territorio nacional. Comparte así su experiencia:

Me interesé por la arteterapia cuando estaba en mi último año de carrera en Arte en la universidad. Tuvimos una experiencia con un compañero que comenzó a llegar a las clases. Él era extranjero, nos dimos cuenta que cursaba su cuarto año de Medicina en otro país, pero se había venido para Costa Rica. A nosotros, los compañeros de último año, nadie nos dijo nada sobre su llegada, era como un secreto. Él empezó a llevar las materias prácticas y parecía que algo le hacía bien, comenzó a sentarse en los recreos con nosotros. Ahí fue donde nos contó que estudiaba allá y que había tenido un problema de salud, no nos dijo qué era. Luego comentó, que el decano de la escuela de Medicina le dijo que no podía continuar en la carrera, que tenía que salirse; eso sería ilegal para este momento. El médico de él, le sugirió que viniera a estudiar Arte en Costa Rica. Le habían dicho que no se preocupara por las notas y que no llevara materias teóricas; que sólo llevara las clases prácticas. A lo largo del semestre él fue mejorando y mejorando. Ahí es donde entra mi mamá quien es psicóloga, Ana Isabel Mora, así se llama. Yo le contaba sobre este compañero y ella me habló sobre arteterapia; me dio una lectura sobre el tema (A. Bulgarelli Mora, comunicación personal, 21 de marzo de 2014).

Agrega Bulgarelli que el segundo semestre, él logró convencer al director de la carrera, que le permitiera asistir a las materias teóricas; el director aceptó. Pero el muchacho empeoró; posiblemente existían otros factores. Después del viernes de la primera semana del segundo semestre, no se le volvió a ver nunca 
más. Señala la entrevistada que cuando empezó a estudiar arteterapia, pensó y pensó en él, que podía haber sido un brote esquizofrénico y problemas de adicciones; es una hipótesis. También recuerda que pintaba precioso, tenía un colorido como el de Matisse (A. Bulgarelli Mora, comunicación personal, 21 de marzo de 2014).

Durante la década de los noventas, Bulgarelli realizó trabajos con niñas, niños y adolescentes. Utilizó la arteterapia a través de experiencias creativas en su práctica privada y en dos instituciones educativas. De marzo de 1993 a setiembre de 1996, se desempeñó como orientadora de primaria en la escuela Country Day School en Escazú y de setiembre de 1996 a marzo del 2002 trabajó en el Colegio María Auxiliadora en San José. Bulgarelli Mora también formó parte de tres equipos clínicos. De setiembre del 2003 a noviembre del 2008, trabajó en la Clínica de Adolescentes del Hospital Nacional de Niños, dirigida por el Doctor Alberto Morales Bejarano. Ángela diseñó e implementó programas de Arteterapia grupal para adolescentes que habían sido diagnosticados/as con trastornos de conducta alimentaria y del estado de ánimo. De abril del 2002 a enero del 2003 colaboró en Villa San José, centro privado para el tratamiento de adicciones. Desarrolló un programa de arteterapia para personas en rehabilitación, residentes en el centro. De abril a diciembre del 2003, formó parte del equipo de la Clínica Integral Especializada en Trastornos Alimenticios, localizada en el Hospital Cima en San José; ahí realizó sesiones individuales y grupales. El 5 de enero del 2004, abrió su Taller de Arteterapia en la comunidad de Tres Ríos Cartago, donde ha realizado sesiones arteterapéuticas con diferentes poblaciones: niños/as, adolescentes, adultos/as y adultos/as mayores. Para el 2007, obtuvo un segundo título de maestría, intercalando su práctica de arteterapéutica con la enseñanza del inglés (A. Bulgarelli Mora, comunicación personal, 21 de marzo de 2014).

En abril del 2008, sucede un acontecimiento muy importante: Ángela Bulgarelli realiza su primer "Curso teórico práctico de aproximación a la arteterapia". Este es avalado por el CENDEISSS y es patrocinado por la Clínica de Adolescentes del Hospital Nacional de Niños. Este curso es el primero, de muchos que vendrán después, para enseñar principios de arteterapia a otros/ as profesionales costarricenses. El Hospital Nacional de Niños se convierte en la cuna de estas primeras capacitaciones. De setiembre a noviembre del 2008 se imparte el curso por segunda vez, en esta ocasión en la Asociación Contra el Cáncer Infantil; de abril a julio del 2009 es el Servicio de Psiquiatría y Psicología del mismo hospital. Gómez Abarca, actual coordinadora del área de Psicología del Servicio de Psiquiatría y Psicología del Hospital Nacional de Niños comenta:

Hicimos un grupo lindísimo. Psiquiatras, psicólogos, todo el equipo, participando en el taller de arteterapia. Fue una experiencia muy linda; en el primer semestre vimos la parte teórica y luego técnicas prácticas. Teníamos que llevar un cuaderno con cada chiquito, uno trabajaba con un chiquito. Ir haciendo un proceso de arte con el 
niño. Entonces se usaba el collage, se usaban recortes, teníamos que hacer un proyecto de graduación; también hacíamos los mandalas; al final ese fue el cierre del taller: hacer un mandala entre todo el grupo. Fue una experiencia muy linda porque como que sale uno de lo tradicional; porque algo que me quedó grabado, es que no todo se puede decir con palabras; sobre todo lo que es trauma, dolor y experiencias vividas por niños que son pequeños, el apoyo de todo lo que es lenguaje no verbal ayuda muchísimo (A. Gómez Abarca, comunicación personal, 10 de abril de 2014).

Desde el 2008 hasta la actualidad, Bulgarelli Mora ha impartido su curso de Aproximación a la Arteterapia en 12 ocasiones. Ella afirma que uno de sus principales objetivos ha sido que los/as estudiantes, aprendan a utilizar el arte plástico como medio terapéutico, agente de estimulación, herramienta de evaluación y lenguaje complementario. Después del Hospital Nacional de Niños, las siguientes sedes para estas formaciones han sido la Clínica Bíblica, la Universidad Católica de Costa Rica, el Centro Cultural Kaleidoscopio Artístico y Natural, la Escuela Casa del Artista del Museo de Arte Costarricense y el CPPCR (A. Bulgarelli Mora, comunicación personal, 21 de marzo de 2014).

De acuerdo con Bulgarelli Mora, el primer curso en las instalaciones del Colegio Profesional de Psicólogos se lleva a cabo de setiembre a diciembre del año 2012. Es fundamental considerar que es el primer colegio profesional de agremiados en Costa Rica, en apoyar directamente la enseñanza de esta disciplina. De febrero a mayo del 2013 se realiza por segunda ocasión y de marzo a junio del 2014 se efectúa por tercera vez; es esta la duodécima versión en términos generales. En agosto del 2013, el CPPCR es sede del primer curso titulado "Introducción a la Arteterapia"; para un total de 13 capacitaciones en la carrera profesional de Bulgarelli Mora. Miranda Orozco, encargado actual del área de capacitaciones del CPPCR, comenta esta experiencia:

Por ahí empecé a conocer de arteterapia, aplicada más al trabajo comunitario, a la psicología comunitaria. Yo visualizaba que tenía una aplicabilidad tangible y concreta. Y digo esto porque los psicólogos podemos, en lugar de entrar en esta discusión de: ¿Qué es psicología? y ¿Qué no es psicología? y ¿Si es algo típico o no? Pues darle una oportunidad y que sean los profesionales quienes tomen la decisión de si esto les sirve o no. Ya que, lo que interesó, es que arteterapia era un enfoque práctico, muchas técnicas... iY esto era lo que venía reclamando el profesional! Entonces empecé a buscar. Por supuesto yo hago revisiones bibliográficas, algún estado de la cuestión en Costa Rica (J.C. Miranda Orozco, comunicación personal, 5 de mayo de 2014).

La labor de Miranda Orozco, tiene un papel clave en la apertura y el apoyo del CPPCR. Durante la misma entrevista él explica: "Lo hicimos de una manera muy supervisada porque sabía que era un tema delicado. iY bueno! Hoy por hoy, sabemos que es un curso regular del colegio. La gente espera todos los años la edición del curso de arteterapia" (2014). Siguiendo a Bulgarelli Mora, Melisa Lobo Chaves jugó también un rol importante; pues ella fue el primer puente de 
comunicación entre Miranda Orozco y su persona (A. Bulgarelli Mora, comunicación personal, 5 de mayo de 2014).

En cuanto a las experiencias universitarias, la arteterapeuta ha impartido distintas clases en universidades públicas y privadas del país. La primera fue en el año 2000 en la Universidad Santa Paula; el curso recibió el nombre de "Medios Terapéuticos IV" y estuvo dirigido a estudiantes de la licenciatura en Terapia Ocupacional. Posteriormente enseñó en la Universidad Nacional, el Instituto Tecnológico de Costa Rica, la Universidad de La Salle, la Universidad Continental de las Ciencias y las Artes, la Universidad Latina de Costa Rica y la Universidad Autónoma de Centroamérica (A. Bulgarelli Mora, comunicación personal, 21 de marzo del 2014).

En Costa Rica, recientemente se empezaron a ofrecer dos diplomados en Arteterapia, uno por la Universidad Centroamericana de Ciencias Sociales (UCASIS, 2014), realizado en conjunto con la Universidad Autónoma Metropolitana (UAM)- Unidad Xochimilco México y el otro, por el Gestal Art Therapy Centre Australia (ARTETERAPIA GESTALT, 2014). Ahora, cabe preguntarse: ¿Representan estas propuestas a nivel educativo, el inicio de posibles escuelas arteterapéuticas en el país? ¿Constituye la labor pedagógica de Ángela Bulgarelli Mora, en conjunto con estos dos diplomados, los primeros pasos para incluir con mayor fuerza la arteterapia en los currículos universitarios costarricenses? Cada vez son más los esfuerzos y los proyectos; un ejemplo de esto es que en setiembre del 2011, se celebró en la sede de la Universidad Católica de Costa Rica, el primer Encuentro Nacional de Arteterapia.

\section{Explorando nuevas posibilidades: investigaciones en universidades}

Visibilizar las primeras investigaciones relacionadas con arteterapia, es fundamental al hablar del desarrollo de este campo de conocimiento en Costa Rica. Estos trabajos representan creación de conocimiento y beneficios para las personas con quienes se laboró. A continuación se presentan las primeras investigaciones científicas relacionadas con arteterapia, tuteladas por Bulgarelli Mora; se aclara que podrían existir trabajos dirigidos por otros/as profesionales, que no se estén incluyendo en el presente artículo. Los trabajos expuestos corresponden a proyectos de graduación de grado y posgrado de profesionales que en su condición de estudiantes, han colaborado a través de la investigación y la puesta en marcha de sus ideas.

En el año 2004, Madrigal Quirós y Rodríguez Esquivel, ambas estudiantes de Psicología de la Universidad Fidélitas, presentan su trabajo final de graduación llamado "Estudio y diseño de un compendio psicoeducativo de actividades terapéuticas basadas en la arteterapia para el abordaje interdisciplinario en el 
tratamiento ambulatorio de las personas diagnosticadas con anorexia nerviosa." Según Madrigal Quirós, el objetivo de la investigación consistía en complementar el abordaje interdisciplinario para el tratamiento ambulatorio de las personas diagnosticadas con anorexia nerviosa; ello, mediante una herramienta psicoterapéutica basada en la arteterapia (K. Madrigal Quirós, comunicación personal, 25 de marzo de 2014). Brenes Vásquez y Lobo Chaves, estudiantes de Psicología, presentan en la Universidad de Costa Rica (UCR) en el 2009, su tesis titulada "Autoexpresión desde la arteterapia: un estudio exploratorio con un grupo de ocho adolescentes de la comunidad Pueblo Nuevo en Pavas". Lobo Chaves comenta:

Con nuestra investigación, queríamos ver los alcances que podía tener la utilización de técnicas arteterapéuticas en un grupo de chicos, en virtud de sus necesidades humanas fundamentales, basadas en una teoría de Max Neef. Max Neef es un economista chileno. En los resultados encontramos la posibilidad que el arte tenía de expandirlos frente a un espacio de hacinamiento. Ellos decían que con el arte les daba como una sensación de expansión y estaban trabajando ahí mismo, no era que nosotras los llevábamos a otra parte. Luego hablaron de la posibilidad de conocerse a sí mismos a través del arte y no sólo de qué le gusta a la persona y qué no le gusta, además de conocer sus facetas, sus diferentes partes. Ellos decían: "...y preguntarnos de cosas que nunca antes nos habíamos preguntado, ni nadie nos había preguntado". "Cuestionarse" fue la palabra más importante y nosotras lo recalcamos mucho. Despertar la capacidad de cuestionar, eso fue algo que todos los chicos que hicieron arteterapia dijeron: "nos comenzamos a preguntar cosas que nunca antes nos habíamos preguntado" (M. Lobo Chaves, comunicación personal, 31 marzo de 2014).

Un año después, en el 2010, Baquero Cassab estudiante de Psicología de la Universidad Latina de Costa Rica (ULatina), expone su trabajo denominado "Arteterapia y logoterapia, propuesta de un modelo de intervención en drogodependencias: Una aproximación humanista basada en los estadios de cambio". Ella explica que su proyecto "buscaba determinar si un proceso estructurado de arteterapia, podía lograr una movilización en el cambio hacia dejar el consumo de drogas". Actualmente se encuentra realizando su tesis de maestría, también relacionada con arteterapia (A. Baquero Cassab, comunicación personal, 28 de abril de 2014).

Bugarelli Mora menciona que para el año 2012, Víquez Brenes presenta su tesis para optar por el grado de máster en Psicología Educativa en la UCR. El nombre del proyecto fue "Autoexpresión en arteterapia para la prevención en adicciones en un colegio semiprivado del área metropolitana - Programa educativo en habilidades sociales". El proyecto de licenciatura de Víquez Brenes versó también sobre la arteterapia. En este mismo año, siguiendo a Bulgarelli Mora, las entonces sustentantes Arce Chaves, Bolaños Torres y Céspedes Rivera exponen el trabajo final de graduación titulado "Técnicas de estimulación temprana para el fortalecimiento del vínculo afectivo entre madre e hijo (a) durante el periodo 
prenatal". Las autoras culminaban su Maestría en Estimulación Temprana en la Universidad Santa Paula. Estas autoras presentaron la arteterapia como parte de su trabajo. De acuerdo con Bulgarelli Mora, una de las integrantes de este grupo, Céspedes Rivera, se convierte en pionera del empleo de la arteterapia en parejas durante el embarazo (A. Bulgarelli Mora, comunicación personal, 5 de mayo de 2014).

En el mismo año, Chaves Salas, Espinach Beirute, Mora Lizano y Pérez Artavia presentan en la escuela de Psicología de la Universidad Católica de Costa Rica, el seminario "Arteterapia como medio terapéutico aplicado a cuidadores domiciliarios de pacientes geriátricos con condición de vida limitada". De acuerdo con Pérez Artavia, trataban de determinar el impacto de la arteterapia en el mejoramiento de la calidad de vida de un grupo de cuidadores domiciliares (S. Pérez Artavia, comunicación personal, 3 de mayo de 2014).

Por último Gutiérrez Chavarría, expone en el 2012, el proyecto "Arteterapia en la promoción de la salud mental en adolescentes en riesgo social" en la Universidad Continental de las Ciencias y el Arte (E. Gutiérrez Chavarría, comunicación personal, 14 de mayo de 2014).

En el año 2013, Calvo Ramírez presentó en la Escuela de Trabajo Social de la Universidad Libre de Costa Rica (ULICORI), su proyecto de graduación "Dimensión socioterapéutica desde la arteterapia para fomentar la cultura de paz: Análisis exploratorio con diez niños y niñas que cursan cuarto grado de la Escuela Quince de Setiembre en el año 2012". En este mismo periodo, Scoh Sotrrell culmina y expone en la Universidad de Iberoamérica (UNIBE) su proyecto "Poniendo colores al camino de la felicidad. Arteterapia y estrategias de afrontamiento: una experiencia con mujeres víctimas de violencia doméstica" (A. Bulgarelli Mora, comunicación personal, 28 de abril de 2014). Finalmente, Castillo Castro y Ugalde Campos, estudiantes de la maestría en Estimulación Temprana de la Universidad Santa Paula, presentan en este año el trabajo "Arteterapia como agente de estimulación temprana en el proceso de vínculo durante el embarazo" (Castillo Castro, comunicación personal, 25 de marzo de 2014).

Para el 2014, se prevé la presentación de dos proyectos más: "Abordaje en niños escolares para desarrollar habilidades intelectuales de innovación y resolución de conflictos con herramientas de la arteterapia", por Núñez Arce y Ramírez Flores; el trabajo se presentará en la Universidad Católica de Costa Rica (S. Núñez Arce \& F. Ramírez Flores, comunicación personal, 8 de abril de 2014).

La segunda, corresponde a: "Manual de estrategias psicopedagógicas para el abordaje del área socio-emocional, mediante técnicas de arteterapia, dirigido a docentes que acompañan el proceso de articulación de niños y niñas entre los niveles de preescolar a primaria", por Garro Sáenz (2014), de la Universidad de La Salle (ULASALLE) (G. Garro Sáenz, comunicación personal, 8 de abril de 2014). 
Recapitulando se señalan varios aspectos relevantes. El primero es, que este apartado corresponde principalmente a sistematizaciones de experiencias; sería interesante por ejemplo, conocer si algunas de ellas han investigado la efectividad del arteterapia. Segundo, todas las investigaciones señaladas son proyectos recientes y han sido realizadas después del año 2000. Tercero, hay personas de distintas carreras, pero en su mayoría han sido estudiantes de Psicología quienes han elaborado trabajos finales de graduación relacionados con el tema. Finalmente, los temas de investigación y las poblaciones con quienes se ha trabajado, han sido muy variadas: adolescentes, cuidadores/as, mujeres embarazadas, estudiantes, docentes, entre otros/as.

\section{Las múltiples caras de la arteterapia}

En Costa Rica, más profesionales se desarrollan en el campo de las "terapias creativas". Se presentan a continuación breves reseñas de otros/as profesionales vinculados con la arteterapia y la psicología. Se esclarece que la presente sección no pretende abarcar la totalidad de los/as involucrados/as, sino presentar una muestra de personas que han sobresalido por sus aportes y que han colaborado con este quehacer en Costa Rica. Bulgarelli Mora (2010) comenta en su sitio web:

Los siguientes son profesionales costarricenses, dedicados a alguna terapia creativa: Lillia Valerio, diplomada en Musicoterapia por el Instituto de Ciencias Sanitarias y de la Educación, de la Pontificia Universidad de Comillas, en Barcelona, España. Laboró varios años en el Hospital Nacional de Niños, con niños autistas y también con niños pacientes del Departamento de OncoHematología. Ella utiliza los sonidos, la música, el silencio y el movimiento en sus sesiones de musicoterapia. Ivania Quesada Chaverri, psicóloga, quien regresó al país hace dos años, después de finalizar su maestría en Danzaterapia, en los Estados Unidos. José Fabio Castillo, músico y profesor en la Universidad Nacional (UNA) en Heredia, quien también se desempeña como musicoterapeuta. Carolina Guier, Psicóloga graduada de la Universidad de Costa Rica, con una Maestría en Terapia Gestalt, de la Universidad Autónoma de Centroamérica. Carolina obtuvo un diplomado en Arteterapia en México.

Guier Acosta, es psicóloga egresada de la UCR. Regresó a Costa Rica en el 2008, luego de estudiar arteterapia en el Centro Integral de Terapia de Arte localizado en la Ciudad de México. Guier Acosta ha desarrollado proyectos en el Hospital Nacional de Niños, ella menciona:

Hice dos trabajos de arteterapia, el primero fue con un grupo de niños y adolescentes, en la Clínica de Adolescentes del Hospital Nacional de Niños, fueron como 4 meses de trabajo. El segundo fue un trabajo en la escuela del Hospital. Se hicieron talleres con el personal de la escuela, casi todas eran profesoras. (C. Guier Acosta, comunicación personal, 8 de abril de 2014). 
Ventura Pozuelo, también psicóloga, terminó su maestría en arteterapia en George Washington University. Trabajó por un año en un centro de rehabilitación de drogas Clean and Sober Streets y en el hospital psiquiátrico Northern Virginia Mental Health Institute, dirigiendo sesiones individuales y grupales de Arteterapia. En Costa Rica, su trabajo final de graduación para optar por el grado de licenciatura, consistió en un proyecto arteterapéutico con adultos/as mayores. Posteriormente estuvo haciendo un voluntariado en el Hospital Nacional de Niños por un año, trabajando con niñas y niños en tratamiento de hemodiálisis y con otros/as víctimas de abuso sexual y físico. En el Albergue de Cristo Rey, laboró aplicando teorías de arteterapia. La referida indica:

Actualmente trabajo en una clínica (GWU Art Therapy Clinic) a nivel individual con pacientes que sufren de estrés postraumático. Considero este enfoque mucho más poderoso que terapia hablada, ya que el componente no verbal, lo hace un tratamiento efectivo para trabajar trauma. Al enfocarse en representar pictóricamente las memorias, los clientes logran acceder y eventualmente integrar memorias fragmentadas en una narrativa que puede llegar a ser asimilada. En otras palabras, como arteterapia involucra todo el cerebro, permite una integración de hemisferios, que puede constituir una experiencia de reestructuración que integra y asimila memorias no verbales.

Cuando regrese a Costa Rica, me gustaría seguir trabajando en colaboración con el Hospital de Niños y eventualmente, desarrollar un programa de arteterapia en el Hospital Nacional Psiquiátrico. También tengo interés en trabajar en clínica privada, odesarrollar mi propio centro de terapias expresivas (M. Ventura Pozuelo, comunicación personal, 31 de marzo de 2014).

Terán Simeone y Valverde Villar, ambos musicoterapeutas, egresados de la Universidad del Salvador en Argentina. Regresaron a Costa Rica en diciembre y agosto del 2012, respectivamente. Actualmente dirigen el proyecto "Musicoterapia Costa Rica". Este tiene como objetivo, difundir y promover la musicoterapia como disciplina de la salud, así como brindar atención clínica y comunitaria (J. Valverde Villar, comunicación personal, 30 de abril de 2014).

En el caso de Valverde Villar, ha desarrollado proyectos de musicoterapia en el Hospital Nacional de Niños, la Asociación Lucha contra el Cáncer Infantil y el Hospital CIMA. Sobre sus charlas en el país se nombran algunos ejemplos: "Cine foro: Mundo Alas. Perspectivas musicoterapéuticas para el abordaje de la discapacidad" el cual se llevó a cabo el 15 de febrero del 2012 en la Escuela de Artes Musicales de la Universidad de Costa Rica; "Mitos y realidades de la musicoterapia" realizada el 13 de octubre del 2012 en la Universidad Santa Paula; "El escenario y la escena vistos desde la Musicoterapia", efectuada el 8 de diciembre del 2012 en el II Congreso Nacional de Psicodrama y "Presentación de la musicoterapia" el 29 de mayo del 2013 en el Hospital Nacional Psiquiátrico (J. Valverde Villar, comunicación personal, 30 de abril de 2014). 
Terán Simeone es parte del equipo de terapia integral del centro privado "Desarrollando Mentes"; trabaja con niñas, niños y adolescentes diagnosticados dentro del espectro autista, asperger y/o algún trastorno generalizado del desarrollo. Es también musicoterapeuta en el Programa de Redes para la Prevención del Menor en Riesgo Psicosocial; aquí realiza intervenciones para trabajar aspectos de violencia, drogas y resiliencia de niñas y niños en condición de marginalidad. Él forma parte del equipo de Musicoterapia del Hospital Nacional de Niños y al igual que Valverde Villar, trabaja en consulta privada desde el proyecto que desarrollaron en conjunto. Menciona:

La musicoterapia como la psicología también tiene sus ramas. Hay musicoterapia conductual, hay musicoterapia analítica, hay musicoterapia psicodinámica, hay musicoterapia gestáltica. La musicoterapia siempre fue pensada muy clínicamente, o clínica, consultorio, mental, psicológico, psiquiátrico o clínica de musicoterapia médica. Pero bueno, en Argentina la cuestión social tiene una importancia muy grande, se empezó a trabajar esta cuestión de la musicoterapia comunitaria. Argentina y Brasil son muy fuertes en esto. Esto es parte de lo que nosotros proponemos con nuestro proyecto: el trabajo con comunidades (M. Terán Simeone, comunicación personal, 31 de marzo de 2014).

Otro caso interesante es el de Viñas Xirinachs, quien es licenciada en Psicología y se desempeña en el ámbito de la danzaterapia. Viñas Xirinachs comenta, que ha sido por más de diez años profesora de danza y que actualmente se forma en el "método Core de Danzaterapia" en "Danzarte: Centro de Danza y Danzaterapia" México. Sobres su quehacer, menciona:

Trabajo como facilitadora en la parte de danzaterapia para un curso de Aproximación a las Terapias Expresivas, en la cual se ven diferentes temáticas y se realizan abordajes desde la danzaterapia, arteterapia y musicoterapia. Otros proyectos realizados, han sido talleres dirigidos a niños. Se trabaja en habilidades sociales, autoestima, expresión corporal, autoconocimiento y expresión de sentimientos (I. Viñas Xirinachs, comunicación personal, 9 de mayo de 2014).

Finalmente, las psicólogas Saskia Salas Calderón y Ginette Sánchez Gutiérrez también han realizado trabajos relacionados con danzaterapia. Salas Calderón, efectuó un proyecto con adolescentes en el Hospital Nacional Psiquiátrico; fue un estudio realizado como tesis de graduación para optar por el grado de Licenciatura en Psicología. A partir de esto, escribe el artículo: "Psicoterapia del movimiento: una propuesta de trabajo en la comunicación de emociones de pacientes esquizofrénicos" (Salas Calderón, 2007). Sánchez Gutiérrez estudia el tango terapéutico, es investigadora de la Maestría en Ciencias del Movimiento Humano de la Universidad de Costa Rica. Junto a Jesenia Hernández Elizondo y a José Moncada Jiménez (2013), presentan el artículo llamado: "Efecto agudo de dos intensidades de tango (baja y moderada) sobre el estado de ánimo de adultos mayores". Mencionan: 
Son muy pocos los estudios sobre el baile del tango en la literatura científica, los pocos encontrados fueron de efecto crónico y mostraron mejorías en el balance en la marcha y beneficios en el estado psicológico y fisiológico en los grupos estudiados luego de varias semanas de práctica. Dado que es una danza, declarada patrimonio de la humanidad recientemente por la UNESCO y practicada por muchas personas alrededor del mundo, incluyendo nuestro país, resulta de interés conocer los factores asociados a su consumo actual como baile social de pareja (p.68).

Después de realizar esta síntesis y de valorar los distintos planteamientos sobre arteterapia, nace la pregunta: ¿La expresión a través de la música, la danza, la poesía, el drama es parte de la arteterapia o al hablar de esta disciplina, se hace referencia únicamente a la actividad plástica? Caruso Galletta (2008), en cuanto a la forma como se le percibe en Argentina, responde:

Nuestro contexto cultural e histórico es diferente del de los países más desarrollados y hegemónicos. No podemos, porque no nos sirve, aplicar aquí de manera no crítica los conocimientos que se desarrollan allá. Quizás es más posible a nivel biológico (una vacuna) que a nivel psicológico (un test), pero menos aún en el nivel sociocultural, que es el ámbito del arte y la terapia.

Tenemos una diferencia fundamental con el criterio anglosajón de arteterapia, que para ellos es exclusivamente referido a la actividad plástica y para nosotros abarca a todas las actividades artísticas (p.227).

Siguiendo a Bulgarelli Mora, la arteterapia, al igual que la danzaterapia, la musicoterapia y la dramaterapia pertenecen a lo que se conoce como "terapias creativas" (A. Bulgarelli Mora, comunicación personal, 5 de mayo de 2014). Las posiciones de ambos responden a contextos distintos en América; para Costa Rica, al ser este un país tan joven en la materia, es relevante conocer las diferentes posturas. Se reconoce riqueza en la diversidad de las voces, pues cada región tiene su propia producción científica y cultural ¿Qué criterio responde mejor a la historia y a la realidad costarricense? ¿Existen otras opiniones?

\section{Discusión: comentarios finales}

¿Cuál es el papel de la psicología en el futuro de la arteterapia en el país? ¿Reunir o separar, vincular o ignorar? El trabajo continuo de diversos profesionales, la apertura constante del Hospital Nacional de Niños para ser un espacio de ejercicio de esta profesión, el reciente apoyo del Colegio Profesional de Psicólogos de Costa Rica (CPPCR) en las capacitaciones, los diplomados, los proyectos de investigación realizados en universidades y el creciente interés de más colegas por tal disciplina, son elementos claves en su porvenir. El contexto costarricense muestra nuevas ideas y nuevos proyectos vinculados a la arteterapia, que podrían ser de interés para los/as psicólogos/as, ¿y por qué no, futuras fuentes de trabajo? 
Tal vez algún día, los hospitales, las escuelas, las organizaciones laborales, las municipalidades, abran espacios cotidianos para este campo de conocimiento y actuación. Por ahora es básico preguntar, ¿qué pasaría si las escuelas de Psicología de las universidades públicas y privadas costarricenses, se interesaran con mayor fuerza por la materia? Young-ha Kim, mencionó en un discurso que dio en Seoul para el reconocido proyecto contemporáneo TED global conference, de la Organización No Gubernamental Sapling Foundation:

¿Por qué la gente se resiste instantáneamente a asociarse con el arte? Quizás piensan que el arte es para la gente muy inteligente o para los que han recibido una buena formación profesional. Y algunos quizá crean que se han alejado demasiado del arte. Puede que sea cierto, pero yo no lo creo. Este es el tema de mi charla hoy. Todos somos artistas natos ("Sé un artista iAhora mismo!", 2010).

Más adelante sostiene: "Tenemos que empezar a crear nuestro propio arte, inmediatamente. Ahora mismo, podemos apagar la tele, cerrar la sesión de Internet, levantarnos y empezar a hacer algo" ("Sé un artista iAhora mismo!,", 2010).

Arteterapia, esa hierbita verde que es capaz de abrirse camino entre las piedras y las tierras áridas.

\section{Referencias bibliográficas}

Adorno, Th.W. (1970). Teoría Estética. Recuperado de https://docs.google.com/ file/d/0B6F7Eoeev69vMmppYjdiTFI5YVU/edit

ARTETERAPIA GESTALT (2014). Formación Arteterapia. Recuperado de http:// www.arteterapiagestalt.es/.

Bonilla Elizondo, P. (2012). La enseñanza del arte en el contexto social actual. Innovaciones educativas, 14(19), 1-10.

Bulgarelli Mora, A. (2010). Terapias Creativas. Recuperado de: http:// arteterapiacostaricaangelabulgarelli.blogspot.com/search?updated$\min =2010-01-\quad$ 01T00:00:00-08:00\&updated-max=2011-01-01T00:00:0008:00\&max-results $=3$

Caruso Galletta, C. (2008). Presentación de la Asociación Argentina de Arteterapia. Arteterapia. Papeles de Arteterapia y Educación Artística para la Inclusión Social, 3, 225-230.

Caruso Galletta, C. Ed. (2011). Silbando en la oscuridad. Música y psicosomática. Buenos Aires, Argentina: Akadia Editorial. 
Ciornai, S. y Diniz, L. (2008). Arteterapia en Brasil. Arteterapia. Papeles de arteterapia y educación artística para la inclusión social, 3, 13-16.

Farias, A. (2007). Arteterapia en Argentina: una historia, un relato, una versión. Arteterapia. Papeles de Arteterapia y Educación Artística para la Inclusión Social, 2, 199-202.

Hogan, S. (2001). Healing Arts: The History of Art Therapy. Recuperado de: http:// S Hogan-2001 - books.google.com

Salas Calderón, S. (2007). Psicoterapia del movimiento: herramienta terapéutica con pacientes esquizofrénicos. Actualidades en Psicología, 21(108), 97-115.

Sánchez, G., Hernández, J., Moncada, J., (2013). Efecto agudo de dos intensidades de tango (baja y moderada) sobre el estado de ánimo de adultos mayores. Actualidades en Psicología, 27(115), 55-70.

Peral Jiménez, C. (2012). El arte y la persona. Arteterapia: esa hierbita verde. Arteterapia. Papeles de arteterapia y educación artística para la inclusión social, 2, 311-312.

Pérez Yglesias, M. (2012). El arte y Francisco Alvarado Abella: Entre la polémica y la crítica. Káñina Revista de Artes y Letras, 36(1), 233-252.

¿Qué es el arteterapia? (2014). Recuperado de http://www.arteterapiachile.cl/ portal/index.php?option=com content\&view $=$ article\&id $=20 \&$ Itemid $=27$

Rubin, J.A. (1999). ART THERAPY: An Introduction. Recuperado de http:// JARubin-1999- books.google.com

Sé un artista iAhora mismo! (2010). Recuperado de http://www.ted.com/talks/ young ha kim be an artist right now/transcript?lang=es

Serrano Morales, P. (2008). Desarrollo del arteterapia en Chile. Arteterapia. Papeles de arteterapia y educación artística para la inclusión social, 3,17-22.

UCASIS (2014). Diplomado en Arte Terapia. Recuperado de http://ucacis.ac.cr/ psicologia/diplomado-arte-terapia.php

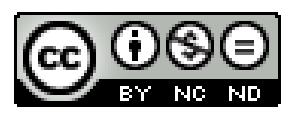

Este obra está bajo una licencia de Creative Commons ReconocimientoNoComercial-SinObraDerivada 4.0 Internacional. 